\title{
Meta-Heuristic Approach for Resource Optimization in Mobile Real Time Video Traffic
}

\author{
P Archana \\ VNR VJIET \\ Hyderabad, \\ India
}

\author{
Subhash S Kulkarni \\ PESIT-South Campus \\ Bangalore, \\ India
}

\begin{abstract}
With the advent of Next generation wireless networks such as $4 \mathrm{G}$, thousands of users will be able to share, create and access live video streaming with different content and characteristics, such as cricket matches and video surveillance using handheld mobile devices. Such services demand new mechanisms for assessing the quality levels of videos. It is imperative for the network operator to exercise stringent control over network parameters and deliver real time video with uncompromising quality in spite of hostile mobile environment. In this paper, Simulated Annealing is used as one of the meta-Heuristic approach for resource optimization in mobile real time video traffic. Other meta-heuristic methods such as Tabu search and genetic algorithms were used in the past. It is found that tabu search gets trapped in local minimum and genetic algorithms are computationally intensive. In this direction, simulated annealing has been found to exhibit effective global minimization with reduced number of iterations. Improvement in performance has been depicted through function plots such as Best function value, Best point, stopping criteria and temperature plot. The algorithm has been tested on standard video quality database available on university at Texas portal.
\end{abstract}

\section{General Terms}

Meta Heuristic, Optimization.

\section{Keywords}

Quality of Experience, Mean opinion score, Pseudo subjective quality assessment, Heuristic method, Simulated Annealing.

\section{INTRODUCTION}

With the Proliferation of Next generation wireless technology coupled with high end mobile computing devices, there can only be an upsurge in multimedia traffic over the coming days. The present day mobile devices are much more than just phones. They are like little mobile PCs, as they provide most of the key features of a personal computer, such as a virtual keyboard with a touch screen, Internet services such as email, web browsing and local Wi-Fi connectivity, built-in camera with image/video capturing, media player etc. In the near future, they may have Mobile TV support for watching live TV programs, networked 3D games and live 3D scene rendering along with high quality visuals. The growth of video traffic is evident from the figure 1.

Some of these features cannot be supported due to much reason, such as the computational demands, power constraints of the mobiles, the available bandwidth and transmission efficiency of the wireless network, the quality of service (QoS), and the compression and error control efficiency of video and graphics data. In this context, there is a need for new mechanisms for determining the quality level of videos as operators are seeking to control their delivery process and optimize their network resources, while increasing the user's satisfaction. Meta-Heuristic algorithms are preferred for solving many network design problems as they provide an optimal solution in a reasonable amount of time. Furthermore, the use of conventional modeling methods demands rigor, precision and certainty, which carry computational cost. On the other hand, heuristic method performs computations, reasoning and inference to reduce computational cost by exploiting tolerance for imprecision, uncertainty, partial truth and approximation.

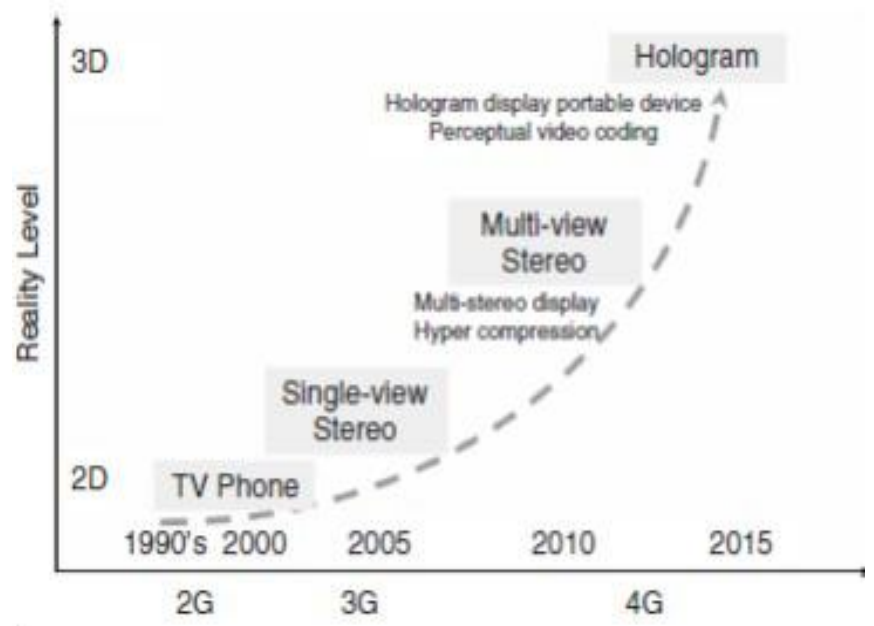

Fig 1: Estimated reality video over wireless development roadmap

Some of the available Meta-Heuristic algorithms [1] are Tabu search (greedy algorithms), Simulated Annealing and Genetic (Evolutionary) algorithms. In this paper Simulated Annealing is used as it is found to exhibit effective global minimization with reduced number of iterations. Tabu search is fast but gets trapped in local minimum and genetic algorithms are computation hungry. In the following sections, rest section deals with issues of real time video transmission over wireless, followed by second section which focuses on video quality assessment based on user's experience. In the third section discussion is on affect of network parameters on transmitted video quality and the next sections deals with optimization of network resources using Simulated Annealing method which is a continuation of the earlier work on video quality assessment [2].

\section{REAL TIME VIDEO OVER WIRELESS}

It is imperative to transmit video and support related real time multimedia applications in the present scenario on the mobile networks, as user`s access Internet through mobile devices. Video applications such as streaming video, video conferencing, online interactive gaming, and mobile TV as 
shown in figure 2 are only a few of the expected applications to support the viability, and survival, of next generation mobile wireless networks. It is therefore necessary to analyze the behavior of the particular medium and its ill effects on the transmitted videos [3].

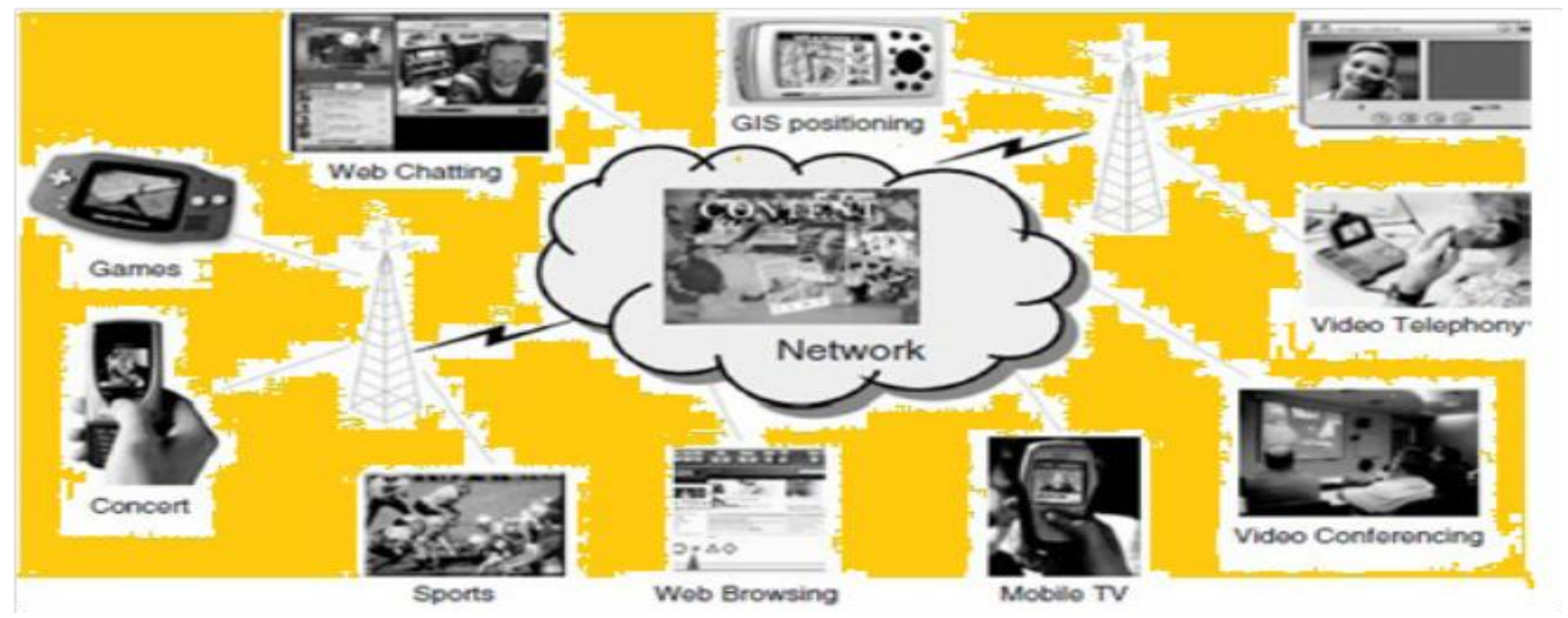

Fig 2: Content has become the center of the network Communications

Several video coding standards as shown in figure 3 have been set up by two organizations, ITU-T and ISO/IEC, including H.26x and the MPEG-x series. MPEG-2/H.262 and MPEG-4 AVC/H.264 are the most common video coding standards. It is required to study the characteristics of mobile wire-less networks and relate these characteristics to the requirements of video transmission, so as to transmit the videos based on the limits of mobile and wireless networks. This helps in the fields of video production and encoding to create videos of high efficiency, in terms of resource utilization, and of high performance, in terms of end-user satisfaction. The relationship derived is based not only on the objective QoS metrics, but also on the subjective quality measures obtained by video viewers at end hosts. Some of the mobile wireless networks are

\subsection{Cellular Wireless Networks}

The cellular $2 \mathrm{G}$ standard called as GSM makes heavy use of digital technology through the use of digital vo-coders, Forward Error Correction (FEC), and high level digital modulation to improve voice quality, security and call reliability. The major drawback of GSM with respect to data and video is that they do not support high data rates. $2.5 \mathrm{G}$ Cellular standard (GPRS) was designed to provide a high data rate packet-switched network. GPRS supports low bit rate video transmission. 3G mobile networks are intended to provide broadband data such as conversational class (voice, video telephony, video gaming), Streaming class (multimedia, video on demand, webcast), Interactive class (web browsing, network gaming, database access) and Background class (email, SMS, downloading) services.

\subsection{IEEE 802.11 WLAN}

Wireless local area networks (WLANs) based on the IEEE 802.11 standard are a significant and viable alternative to wireless connectivity. The standard has many variations such as $802.11 \mathrm{~b}$ which operates at $11 \mathrm{Mbps}$, but operates also on 1 , 2 and $5 \mathrm{Mbps}$. The $802.11 \mathrm{a}$ and $\mathrm{g}$ operate on the $5 \mathrm{GHz}$ and $2.4 \mathrm{GHz}$ bands respectively and both have a maximum theoretical data rate of $54 \mathrm{Mbps}$. The recent IEEE $802.11 \mathrm{e}$ is a multimedia standard.



Fig 3: Video coding standards

\section{VIDEO QUALITY ASSESSMENT METHODS}

The protocols used in the networks are aware of quality of service (QOS) but none takes into consideration the quality of experience (QOE), which is the most important factor in the increasing multimedia traffic such as video and voice applications today. A number of the mechanisms rely on technical parameters, especially bandwidth. Available and requested bandwidths are compared before deciding whether to accept a connection or not, as in resource provisioning approach. This works well with wired networks where bandwidth provisioning is easier than in the wireless environment. In addition, bandwidth alone is not enough to guarantee quality; consequently, other works have been proposed to evaluate quality, for example, Park et al. [4] make use of Peak Signal to Noise Ratio (PSNR), basically used to compare quality of receiving to transmitting videos, to estimate quality. However, PSNR can only be measured once the video traffic has arrived at receivers, so this approach is not appropriate for controlling admission in real-time. To enable high quality for admitted flows and dynamic adaptation to network conditions, QoE based admission control mechanism is used that administers the access network in real-time based on user's perceived quality. 


\subsection{Quality of Experience Assessment}

Generally, traffics are monitored in terms of technical parameters such as throughput, delay, jitter, and loss. These parameters give technical performances of the network but they do not reveal efficiently the QoE perceived by users. In order to evaluate this QoE, several mechanisms have been proposed in both objective and subjective approaches. The former approach consists in measuring QoS by monitoring

Network parameters such as throughput, latency, and error whereas the latter consists in measuring QoE evaluated by real humans in terms of Mean Opinion Score (MOS), as shown in table 1 .

Table 1. MOS Quality Scale

\begin{tabular}{|l|l|l|}
\hline MOS & Quality & Impairment \\
\hline 5 & Excellent & Imperceptible \\
\hline 4 & Good & Perceptible but annoying \\
\hline 3 & Fair & Slightly annoying \\
\hline 2 & Poor & Annoying \\
\hline 1 & Bad & Very Annoying \\
\hline
\end{tabular}

The problem of the objective approach is an inaccuracy of the score; because the score is based only on technical parameters, it does not correlate well with real human evaluation. On the other hand, the subjective approach is accurate but high-cost and time-consuming. This makes it hard to repeat often and it cannot be used in real time. Hence, pseudo-subjective quality assessment is used.

\subsection{Pseudo-Subjective Quality Assessment}

Considering advantages and drawbacks of the previous approaches, to achieve the perceptual quality evaluation, a method called as Pseudo-Subjective Quality Assessment (PSQA) [5], which is hybrid between subjective and objective approaches. It keeps advantages and eliminates drawbacks of both approaches. PSQA is based on statistic learning using random neural network (RNN). The idea is to train the RNN to learn the mapping between QoE score and technical parameters so that a trained-RNN can be used as a function to give QoE score in real-time. In order to use this tool, three steps need to be done a priori are Configuration, Training and Validation.

\section{CHALLENGES IN MOBILE REAL TIME VIDEO TRANSMISSIONS}

A lot of content is being generated and uploaded onto all kinds of networks with various throughputs, quality of services and protocols, and heterogeneous terminals with a wide range of capabilities, accessibilities and user preference. Against such a background of expectations, multimedia access has become a challenge for the Next generation wireless networks. This can be viewed as a mathematical optimization problem for which a variety of algorithms can be applied. In the worst case the problem to be solved is considered as mixed-variable multi criteria optimization problem with general constraints as it is discussed in. Through the work of [6], the quality perceived by the user is obtained through corresponding network parameters to be used in a video transmission system, mainly from the values of the bit rate and of the packet loss within the network, and its maximum value is influenced by the frame rate of the video proportionally. Therefore, the final formula used to obtain the perceived quality of a video streaming is given as follows.

\subsection{Packet Loss Affect}

All the packet loss considered in the videos were assumed to be random losses distributed over an uniform probability density function which means that all the packets have the same probability to be dropped. The video quality function as a result of packet loss is given by equation 1 .

$$
\mathbf{f}_{\mathbf{L}}(\mathbf{l}, \mathbf{b r})=\frac{P 1(b r) \cdot l^{2}+P 2(b r) \cdot l+P 3(b r)}{l+Q 1(b r)} \quad \mathbf{E Q . 1}
$$

where 1 is the packet loss variable, br stands for link bit rate offered by the network. The values of constants P1, P2, P3 and Q1 for the different bit rates are given by Equation 2 . Hence packet loss, bit rate collectively affect the perceived video quality.

\subsection{Bandwidth Affect}

Another parameter that can influence in the video stream quality is the bandwidth. The equations that describe the different constant parameters from Equation 1 are as follows.

$$
\begin{gathered}
P 1(b r)=-0.1387 \cdot e^{2.721 \cdot b r / 10000+} \\
\text { 0.2823. } e^{-8.885 \cdot b r / 1000} \\
P 2(b r)=2.154 \cdot e_{1}^{1.584 \cdot b r / 10000}- \\
\text { 2.125. } e^{-7.8 \cdot b r / 1000} \\
P 3(b r)=1.95 \cdot e_{1}^{2.887 \cdot b r / 10000}- \\
\text { 1.307. } e^{-9.414 \cdot b r / 1000} \\
Q 1=\frac{1.75 \cdot b r^{3}}{10^{10}} \frac{4.327 \cdot b r^{2}}{10^{7}}+\frac{4.19 \cdot b r^{1}}{10^{4}}+0.3876
\end{gathered}
$$

For some of the bit rates, constants P1, P2, P3 and Q1 are given as follows

$$
\begin{aligned}
& \mathrm{f}_{150}(1)=\frac{-0.0694 . l^{2}+1.545 . l+1.719}{l+0.447} \\
& \mathrm{f}_{300}(1)=\frac{-0.1343 . l^{2}+2.061 . l+2.041}{l+0.4721} \\
& \mathrm{f}_{600}(1)=\frac{-0.1591 . l^{2}+2.333 . l+2.342}{l+0.5274} \\
& \mathrm{f}_{900}(1)=\frac{-0.1757 . l^{2}+2.496 . l+2.5}{l+0.5391}
\end{aligned}
$$

\subsection{Frame Rate Affect}

Video quality perceived is also a function of video frame rate. Perceived video quality depends on mainly three parameters: such as motion variations (i.e., cricket match vs. interview), sound and visual content. Based on these parameters, classification of video based on the perception of the users is made. Equation 3 relates video quality to the frame rate.

$$
\mathrm{f}_{\mathrm{R}}(f p s)=\frac{-0.00102 . f p s^{2}+1.164 . f p s+1.704}{f p s+5.714}
$$


The final equation to compute video quality expresses in terms of Mean Opinion Score (MOS) is given by equation 4

$$
\operatorname{MOS}(1, \text { br, fps })=\mathbf{f}_{\mathrm{L}}(\mathbf{l}, \mathrm{br}) * \mathbf{f}_{\mathrm{R}}(\mathrm{fps}) \quad \text { Eq. } 4
$$

\section{META- HEURISTIC APPROACH FOR RESOURCE OPTIMIZATION}

As discussed in previous section, influence of network parameters on perceived video quality can be seen as a multivariant optimization problem. Heuristic algorithms tend to give a good solution in less amount of time; examples on

Heuristic algorithms that give approximate solutions are Simulated Annealing, Tabu Search and Genetic Algorithms (or Evolutionary Algorithms). The idea behind is to provide a recommendation on which of those algorithms performs best for the given problem at hand.

Meta-heuristics [7] are a wide family of algorithms used to solve computationally complicated optimization problems, by computing a cost function that is subject to a set of constraints on the potential solution. The cost function (also called objective function) itself is treated as a black box, and the navigation between various potential solutions (local search) is normally a state transition that is either uphill to downhill respectively for maximization and minimization problems.

\subsection{Simulated Annealing (SA)}

A single point search algorithm, SA is an inspiration from a thermodynamic process where solids are brought to high temperature and then cooled slowly back to the original temperature. The crystalline state reached after such a process has a minimum energy after transiting back from a liquid to a crystal-like structure. The transition from thermodynamics to meta-heuristics is introduced by Kirkpatrik [8]. The following table (Table 2) summarizes the mapping between both fields.

Table 2. Simulated Annealing: Mapping Thermodynamics to Optimization

\begin{tabular}{|l|l|}
\hline Thermodynamics & Optimization \\
\hline System State & Potential Solution \\
\hline State Energy & Cost Function Evaluation \\
\hline Temperature & Iterative Control Parameter \\
\hline State Transition & Neighboring Solution \\
\hline Crystal State & Optimal Solution \\
\hline
\end{tabular}

Equation (5) below can be used for SA implementation where Tactual and Tinitial refer to the actual and initial temperatures, respectively. Paccept is the probability of accepting a solution with a cost function that is worse than the previously evaluated better neighboring solution vector. The flow chart for Simulated Annealing is shown in figure 4.

$\mathrm{P}_{(\text {accept })}=$

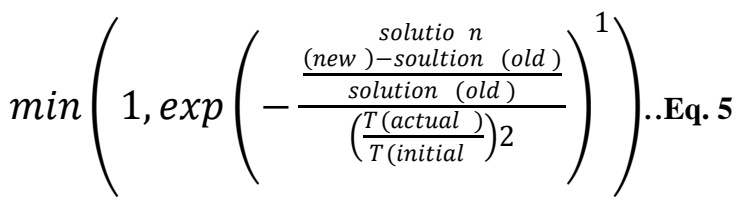

\subsection{Tabu Search}

Another single point search algorithm, Tabu Search differs from SA in that it is not a stochastic method and involves an adaptive memory function. It therefore requires less computational effort and targets adaptation rather than planning. It is suited for combinatorial optimization problems.

The flow chart for Simulated Annealing is shown in figure 5.

\subsection{Genetic Algorithms}

Genetic Algorithms, also known as Evolutionary Algorithms, are inspired from the evolution of populations with the principle that the fittest will survive and thrive. Accordingly, only the best genes are kept and recombined to give birth to individuals that satisfy the cost function. They differ from the

Previous two algorithms in their parallelism as compared to single point search. The flow chart for Simulated Annealing is shown in figure 6 .

\section{RESOURCE OPTIMIZATION USING SIMULATED ANNEALING}

In this paper Simulated Annealing (SA) meta-heuristic method is proposed to provide resource optimization in mobile real time video traffic. SA is considered as it performs well by converging to optimal values in a feasible time for keeping the adaptation in real or near real-time. Tabu search is not preferred as there is possibility of converging to local minimum. Genetic algorithms converge to optimum values gradually with increasing number of iterations, making them rigid for real time situations. The set of equations as given in section 4 are used for searching the optimal solution, by setting the video quality affecting network parameters as in Table 3. These parameters are chosen from the standard mobile video databases.

Table 3. Parameters for Simulated Annealing

\begin{tabular}{|l|l|l|}
\hline Parameter Type & Minimum & Maximum \\
\hline Bit Rate in kbps & 150 & 1500 \\
\hline Packet loss in \% & 0.0 & 0.1 \\
\hline Frame Rate in fps & 5 & 30 \\
\hline
\end{tabular}

The objective function to be minimized is the MOS equation (Eq.4) that relates all the above mentioned parameters to the perceptual video quality.

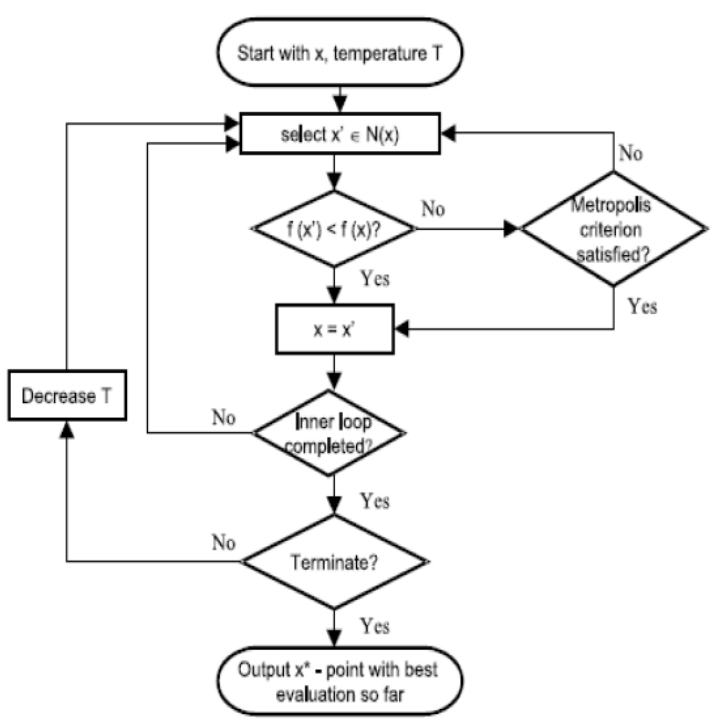

Fig 4: Flowchart for Simulated Annealing 


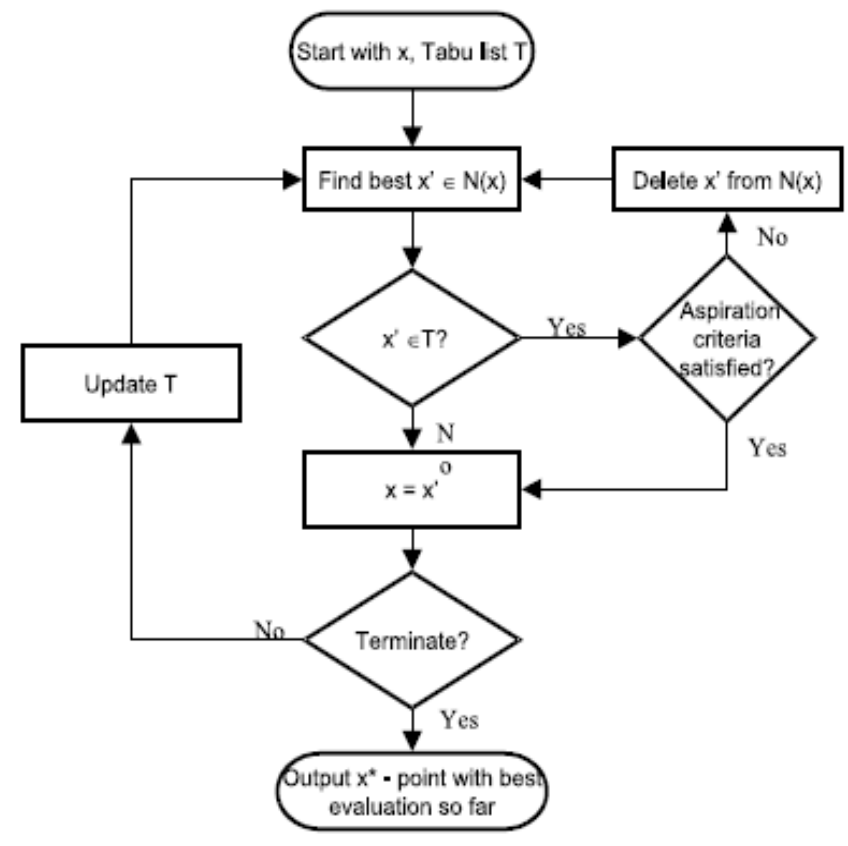

Fig 5: Flowchart for Tabu Search

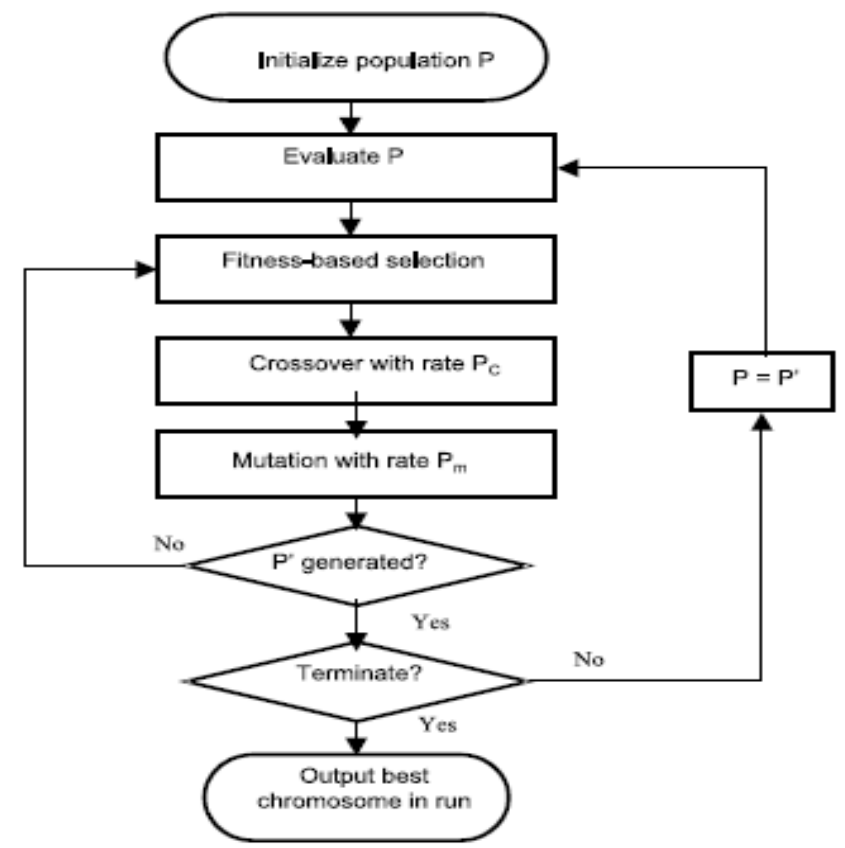

Fig 6: Flowchart for Genetic Algorithm

\section{RESULTS}

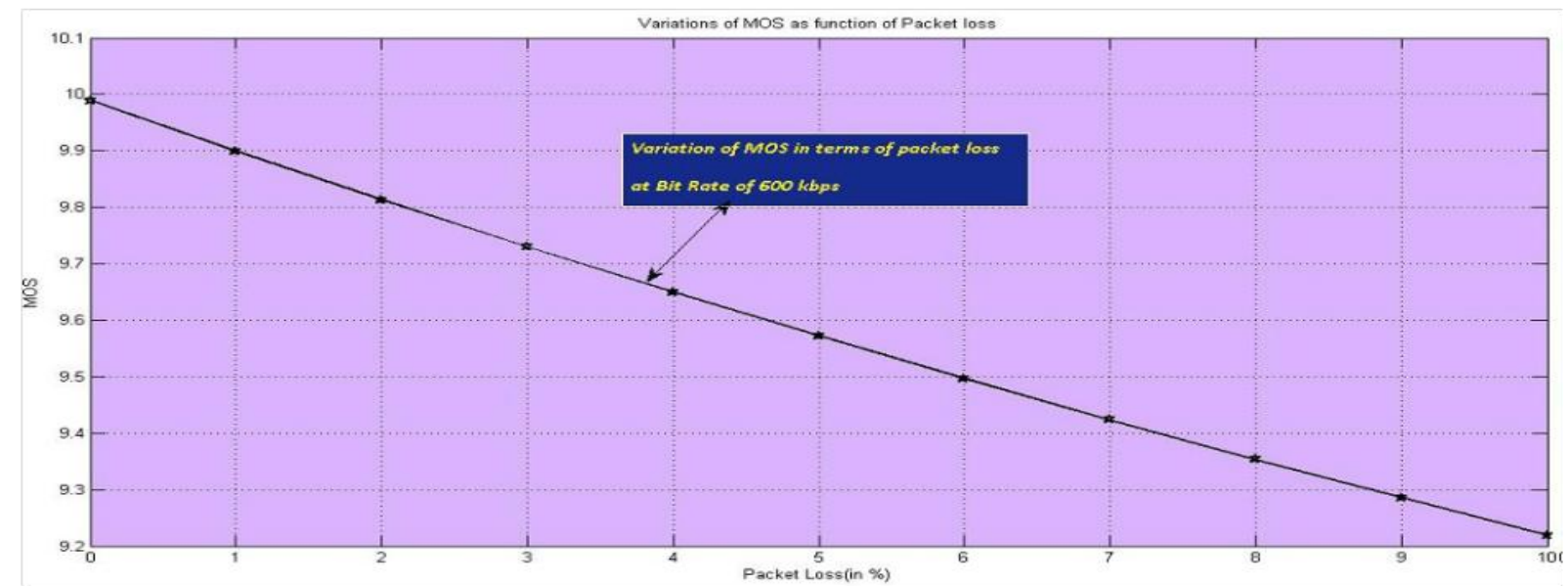

Fig 7: Shows variation of MOS as a function of Percent Packet loss. It can be seen that with increase in packet loss MOS reduces, in turn reducing the QOE indicating low user`s satisfaction

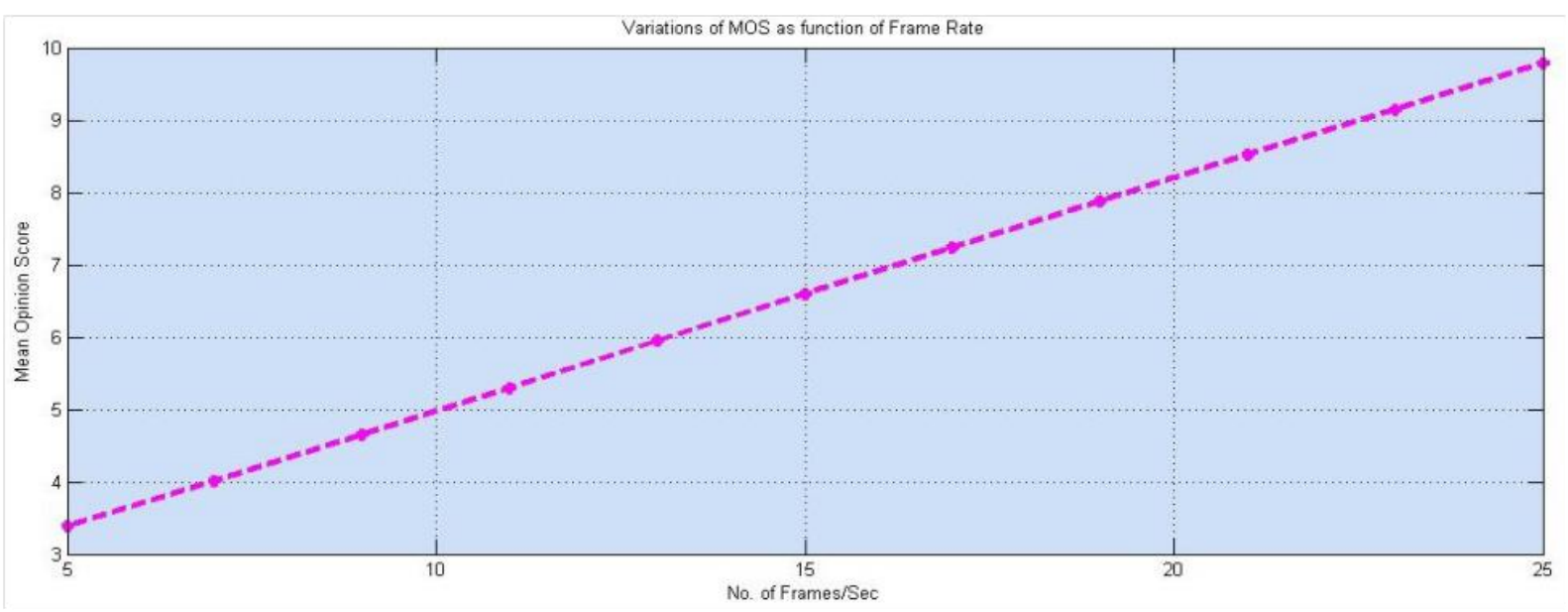

Fig 8: Shows variation of MOS as a function of Frames transmitted per second. It can be seen That with increase in frame rate MOS increases, leading to higher QOE. 


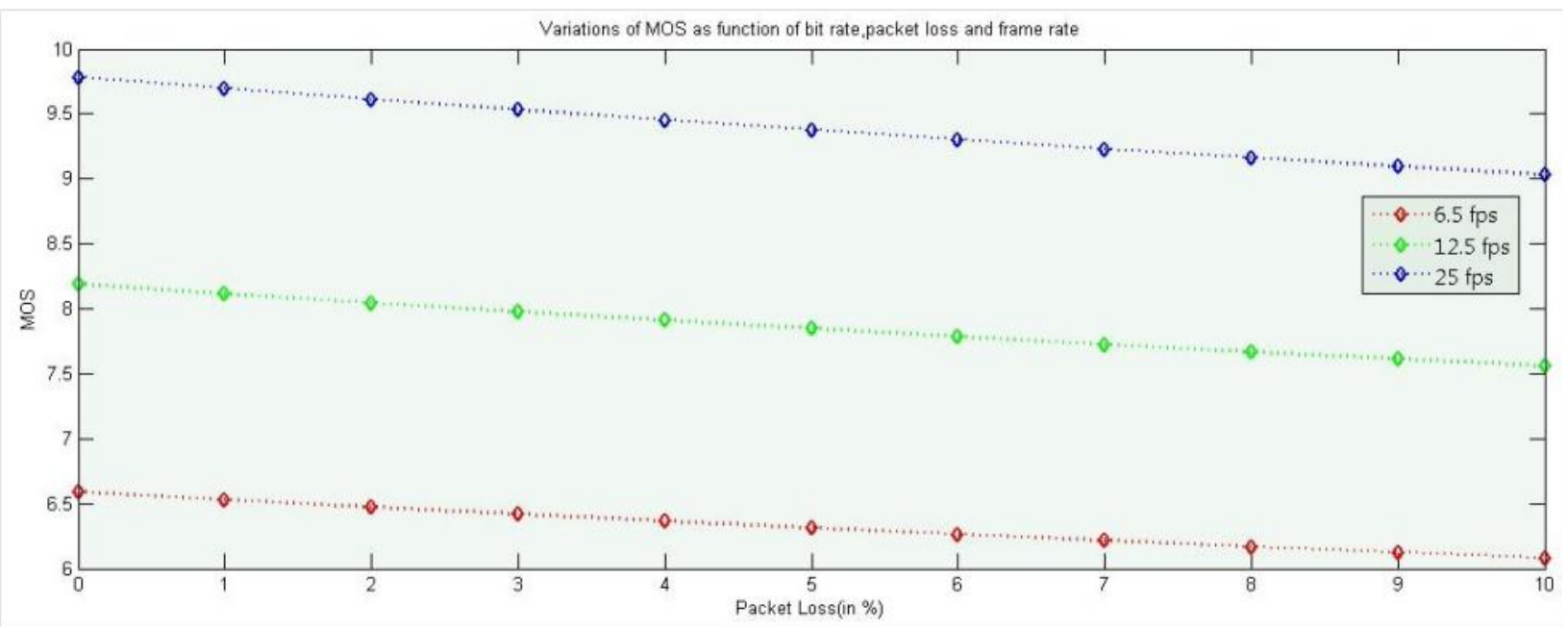

Fig 9: Shows variation of MOS as a function of Percent Packet loss, bit rate and frame rate.
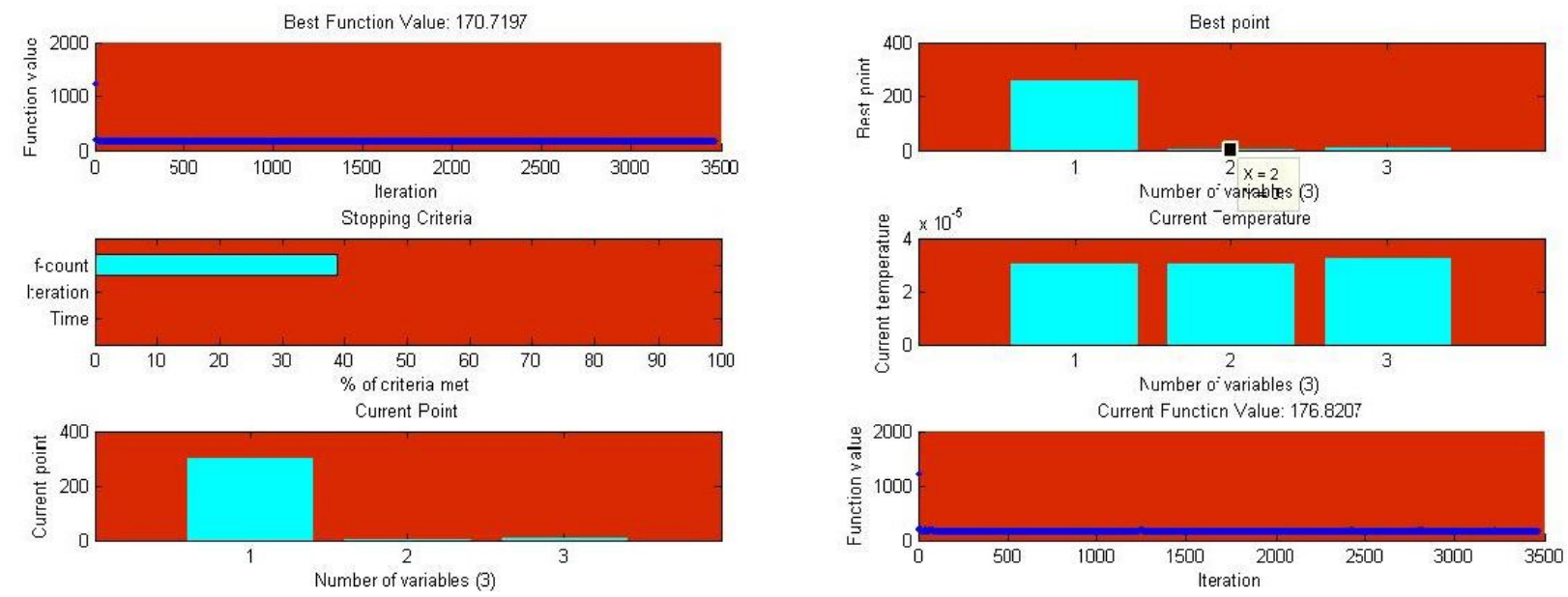

Figure 7: Shows Simulated Annealing performance in terms of Best function value, Best point and Stopping Criteria as function count along with current temperature value, current point, and current function value.


Fig 10: Shows Simulated Annealing performance in terms of Best function value, Best point and Stopping Criteria as function time along with current temperature value, current point, and current function value. 

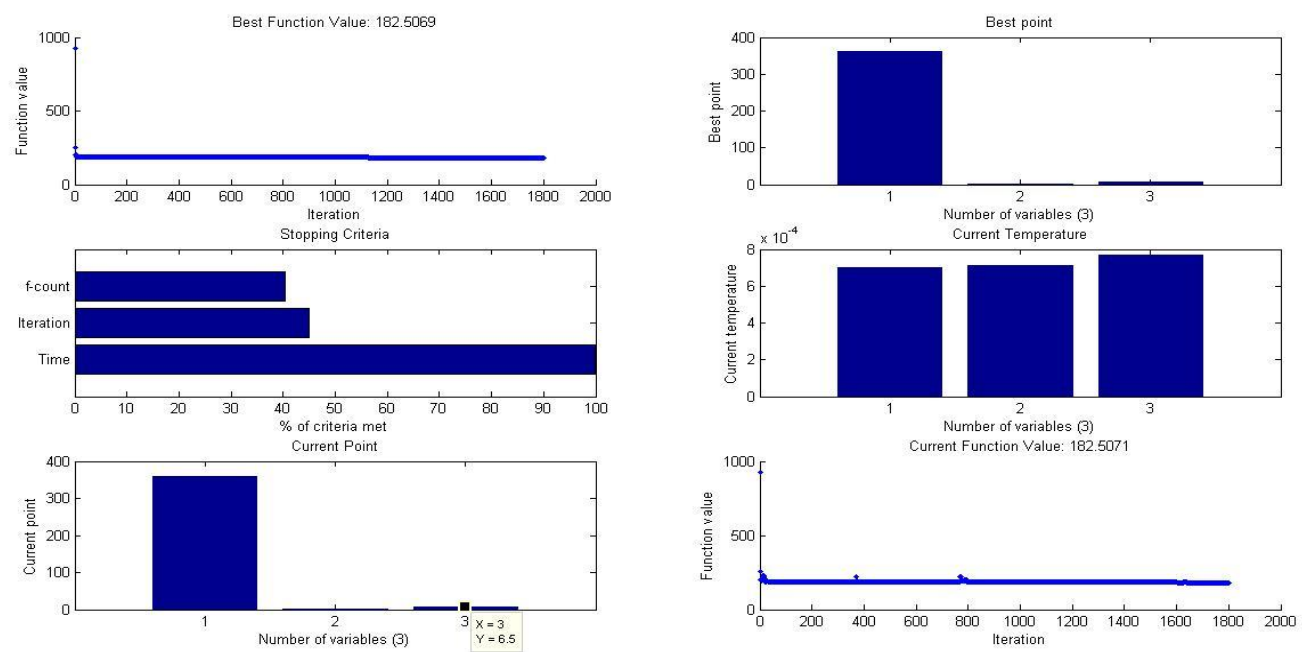

Fig 11: shows Simulated Annealing performance in terms of Best function value, Best point and Stopping Criteria as function time along with current temperature value, current point, and current function value.

\section{CONCLUSIONS}

These results showed that the proposed SA method provides an optimal solution with reduced reasonable complexity. It can be concluded that SA gets to the optimum solution within stipulated time. In future, study of new optimization methods that can provide simplifications of the objective functions used and their conditions for an even faster real-time adaptation such as online optimization would be of worth.

With increasing data rates as promised by $4 \mathrm{G}$ implementation,

There will be a splurge in multimedia data on Internet. Hence, optimizing video transmission affecting parameters becomes

A necessity rather than option. Future scope includes enhancement of QOS protocols based on MOS values for real time video transmissions and also improved QOS based routing protocols in a mobile IP based networks.

\section{REFERENCES}

[1] Miguel Rios, Vladimir Marianov, and Cristian Abaroa. Design of Heterogeneous Tra_c. 2005. Networks Using Simulated Annealing Algorithms. C. Kim (Ed.): ICOIN 2005, Springer-Verlag Berlin Heidelberg 2005.

[2] P. Archana, Subhash Kulkarni, Jan 2014. Real Time Video Quality Measurement Using LevenbergMarquardt Back propagation Algorithm. Fifth International Conference on Signals and Image Processing (ICSIP )

[3] Vasos Vassiliou, Pavlos Antoniou, Iraklis Giannakou, and Andreas Pitsillides, 2006. Requirements for the Transmission of Streaming Video in Mobile Wireless Networks. Artificial Neural Networks - ICANN 2006

[4] Y Park, Y. Seok, N. Choi, j.-M Bonnin.Jan 2006, Rateadaptive multimedia multicasting over IEEE 802.11 wireless LANs. Consumer communications and Networking Conference, 2006. CCNC 2006. 3rd IEEE.

[5] Kandaraj Piamrat, Adlen Ksentini, C.Viho, Jean-Marie Bonnin. . 2008. QoE-aware Admission Control for Multimedia Applications in IEEE 802.11 Wireless Networks.

[6] Christian Timmerer1,Victor H. Ortega, Jose M. Gonzalez, Alberto Leon. 2008, Measuring quality of experience for MPEG-21-based cross-layer multimedia content adaptation; Proceeding AICCSA '08 Proceedings of the 2008 IEEE/ACS International Conference on Computer Systems and Applications.

[7] Miguel Rios, Vladimir Marianov, and Cristian Abaroa. 2005, Design of Heterogeneous Traffic Networks Using Simulated Annealing Algorithms. ICOIN 2005.

[8] S.Kirkpatrick,C.D. Gelatt, and M. P.Vecchi. 1983 Optimization by Simulated Annealing .Science 220(4598):671\{680 (1983)

[9] A book by Shaohong Wang,Lisimachos, P. Kondi, Ajay Luthra, Song $\mathrm{Ci}$, A John. 4G wireless Video communication

[10] Eduardo Cerqueira, Sherali Zeadally, Mikołaj Leszczuk, Marilia Curado, Andreas Mauthe. 2011, Recent advances in multimedia networking Multimed Tools Appl (2011) 54:635-647 DOI 10.1007/s11042-010-0578-z

[11] Abhijit Bijwe, C.G.Dethe. 2013.Vertical Handoff algorithms using Neural networks. Proc. of the Second Intl. Conf. on Advances in Computer, Electronics and Electrical Engineering -- CEEE 2013 ISBN: 978-981-076260-5 doi:10.3850/ 978-981-07-6260-5_10

[12] Elisangela Aguiar, Andre' Riker, Eduardo Cerqueira, Antonio Abelem, $\mathrm{Mu} \mathrm{Mu}$, Torsten Braun, Marilia Curado, Sherali Zeadally. 2014. A real-time video quality estimator for emerging wireless multimedia systems. Wireless Netw.DOI 10.1007/s11276-014-0709y

[13] Aderemi A. Atayero, Matthew K. Luka. 2012. International Journal of Computer Applications (0975 8887) Volume 45- No.22, May 201248 Applications of Soft Computing in Mobile and Wireless Communications

[14] Mohammed A Qadeer, Rehan A hmad, Mohd Siddique Khan, Tauseef Ahmad. 2009. Real Time Video Streaming over Heterogeneous Networks, Feb. 15-18, 2009 ICACT 2009, Page(s): 1117 - 1122.

[15] Lal Chand Bishnoi,Dharm Singh, Shailendra Mishra, Simulation of Video Transmission over Wireless IP Network in Fedora Environment, IP Multimedia Communications A Special Issue from IJCA 